\title{
A retrospective audit to assess the safety and efficacy of apremilast use in chronic plaque psoriasis
}

\author{
Authors: Katie Kwan ${ }^{\mathrm{A}}$ and Jui Vyas ${ }^{\mathrm{B}}$
}

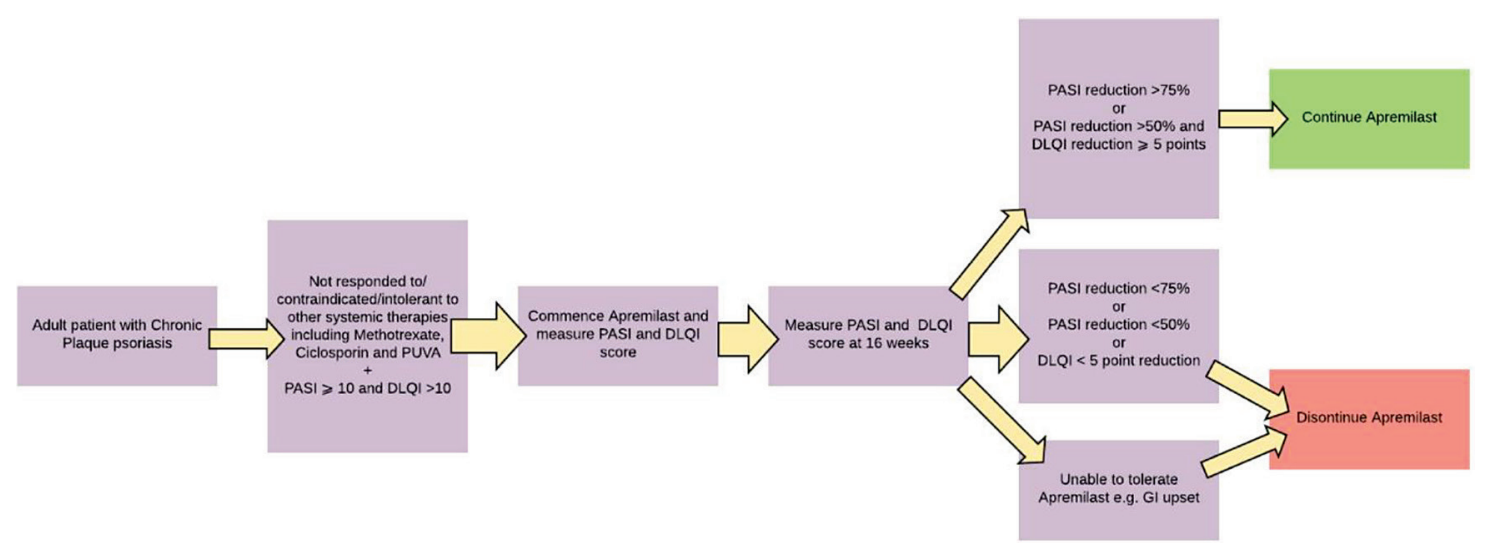

Fig 1. Overview of commencing and stopping apremilast.

\section{Introduction}

Apremilast is a small molecule inhibitor of phosphodiesterase 4 , an intracellular enzyme which modulates the expression of cytokines and mediators including tumour necrosis factor $\alpha$ and interleukin 23. ${ }^{1}$ It was licensed for use by the European Commission in 2015 and, following a recent review, the National Institute for Health and Care Excellence (NICE) has also recommended it as a treatment option for patients with severe plaque psoriasis from November 2016. The purpose of this audit was to assess the safety and efficacy of apremilast use in adult patients with moderate to severe plaque psoriasis.

\section{Material and methods}

Retrospective analysis was conducted using individual patient medical documents and an online clinical portal. Patients prescribed apremilast for the indication of plaque psoriasis between 01 September 2017 - 12 March 2018 were included in the audit $(n=44)$. Two hospitals of a health board in the UK were involved in this study. The gold standard used to compare safety and efficacy of apremilast was the NICE technology appraisal guidance (TA419). ${ }^{2}$ Clinical efficacy of the drug was determined by

Authors: ${ }^{A}$ Cardiff University School of Medicine, Cardiff, UK; ${ }^{\mathrm{B}}$ University Hospital of Wales, Cardiff, UK assessing the Psoriasis and Severity Index (PASI) and Dermatology Life Quality Index (DLQI) scores.

\section{Results and discussion}

Fig 1 displays an overview of commencing and stopping apremilast according to NICE guidance. Findings show that 13 patients (30\%) met the NICE guidance inclusion criteria for the use of apremilast. Twenty-five patients had a PASI and DLQI assessed pre-treatment and 28 patients had exhausted other systemic therapies (or had contraindications to) including methotrexate, ciclosporin and psoralen-UVA therapy. Of those 28 patients, 13 also had a PASI $\geq 10$ and $D L Q I \geq 10$. An inadequate response was determined by calculating the score reduction percentage of PASI and DLQI prior to and following 16 weeks of treatment. In terms of drug efficacy 25 patients $(57 \%)$ responded to apremilast according to medical notes and continued treatment, 16 patients (36\%) discontinued treatment including $14 \%$ due to associated side/adverse effects. Gastrointestinal upset was the most common side effect and $9 \%$ of patients developed new depression during treatment. Three patients had not completed 16 weeks at the end of the audit period.

\section{Conclusion}

Findings show that NICE guidance standard was largely not met with respect to assessing the severity of psoriasis using the PASI and DLQI scores in combination with exhausting alternative 
systemic therapies before considering apremilast. However, medical notes documented improvement in psoriasis from clinician and patient perspectives in responders. Although we found depression in $9 \%$ of patients, it must be noted that this was based on patient views and a validated tool was not used to calculate depression. Advantages to the use of apremilast include its oral administration, minimal drug interaction potential and tolerable side effect profile. Given that treatment of psoriasis is highly individualised, apremilast will likely be of value to patients who cannot tolerate and/or are unresponsive to conventional systemic non-biologic agents. Furthermore, the cost of apremilast ( $£ 555$ for a 28 -day pack after initial titration) is lower than that of biologic therapy and will potentially have a bigger role in treating more patients with psoriasis in the future.

\section{Conflicts of interest}

None declared.

\section{References}

1 National Institute for Health and Care Excellence. APREMILAST. NICE, 2019. [Accessed 27 October 2019] https://bnf.nice.org.uk/ drug/apremilast.html.

2 National Institute for Health and Care Excellence. Apremilast for treating moderate to severe plaque psoriasis. Technology appraisal guidance [TA419]. NICE, 2019. www.nice.org.uk/guidance/ta419 [Accessed 27 October 2019]. 